\title{
Protocolo GDLAM de avaliação da autonomia funcional
}

\author{
Artigo Original
}

\section{Estélio Henrique Martin Dantas,}

PROCIMH - Universidade Castelo Branco / RJ - Brasil

LABIMH - UCB / RJ

Grupo de Desenvolvimento Latino-Americano para Maturidade - GDLAM

Bolsista de Produtividade em Pesquisa - CNPq

estelio@cobrase.com.br

\section{Rodrigo Gomes de Souza Vale}

PROCIMH - Universidade Castelo Branco / RJ - Brasil

LABIMH - UCB / RJ

Grupo de Desenvolvimento Latino-Americano para Maturidade - GDLAM

vale@redelagos.com.br

DANTAS, E.H.M., VALE, R.G.S. Protocolo GDLAM de avaliação da autonomia funcional. Fitness \& Performance Journal, v.3, n.3, p. 175-182, 2004.

Resumo: $\bigcirc$ presente estudo teve como objetivo estabelecer um protocolo de avaliação da autonomia funcional através de testes relacionados com a realização das atividades da vida diária (AVD). A amostra foi constituída de 337 mulheres idosas (C $=66,33 \pm 4,69$ anos), voluntárias e independentes das AVD. Foram utilizados os testes do protocolo de avaliação da autonomia funcional do Grupo de Desenvolvimento Latino-Americano para a Maturidade (Protocolo GDLAM), constituídos de caminhar 10m (C10m), levantar-se da posição sentada (LPS), levantar-se da posição decúbito ventral (LPDV) e levantar-se da cadeira e locomoverse pela casa (LCLC). $\bigcirc$ tratamento estatístico feito por meio de Quartis possibilitou estabelecer o índice GDLAM de autonomia (IG). Este apresentou escores classificados em fraco $(+28,54)$, regular $(28,54-25,25)$, bom $(25,24-22,18)$ e muito bom $(-22,18)$. estudo admitiu o nível de $p<0,05$ para a significância estatística.

Palavras-chave: autonomia funcional, idoso, AVD.

Endereço para correspondência:

LABIMH - Av Salvador Allende, 6700 - Recreio dos Bandeirantes - Rio de Janeiro - RJ - CEP 22780-160 


\section{GDLAM'S protocol of functional autonomy evaluation.}

The present study had the aim to establish a functional autonomy evaluation protocol throughout tests related with the realization of the activities of daily living (ADL). The sample was formed of 275 old women (' $C=66,33 \pm 4,69$ years old), voluntaries and independent on the ADL. Tests of functional autonomy evaluation protocol by Latin-American Development to the Maturity Group (GDLAM'S Protocol) were used. GDLAM'S Protocol was formed for C10m, LPS, LPDV and LCLC tests. The statistical analysis made by Quartiles established the autonomy GDLAM index (IG). The IG showed scores classified on poor $(+28,54)$, regular $(28,54-25,25)$, good $(25,24-22,18)$ and very good $(-22,18)$. The study admitted the level of $p<0,05$ for statistic significance.

Keywords: functional autonomy, old, ADL.

\section{Protocolo GDLAM de evaluación de la autonomia funcional}

El presente estudio hubo como objetivo establecer un protocolo de evaluación de la autonomía funcional a través de testes relacionados con la realización de las actividades de la vida diaria (AVD). La muestra fue constituida de 337 mujeres ancianas ( $C=66,33 \pm 4,69$ años), voluntarias y independientes de las AVD. Fueron utilizados los testes de lo protocolo de evaluación de la autonomía funcional del Grupo de Desenvolvimiento Latino-Americano para la Madurez (Protocolo GDLAM) constituidos de caminar $10 \mathrm{~m}(\mathrm{C} 10 \mathrm{~m})$, levantarse de la posición asentada (LPS), levantarse de la posición decúbito ventral (LPDV) y levantarse de la silla y moverse por la casa (LCLC). El tratamiento estadístico echo por el medio de Quartil posibilitó establecer el índice GDLAM de autonomía (IG). Este presentó escores calificados en flaco $(+28,54)$ regular $(28,54-25,25)$, bueno $(25,24-22,18)$ y muy bueno $(-22,18)$. El estudio admitió el nivel de $p<0,05$ para la significación estadística.

Palabras clave: autonomía funcional, ancianas, AVD.

\section{INTRODUÇÃO}

A expectativa de vida está aumentando e o envelhecimento populacional está ocorrendo em quase todos os países do mundo, principalmente nos países em desenvolvimento (IBGE, 2000). E o que se deseja ao ser humano é que ele seja independente em suas atividades diárias e suas decisões, ou seja, que viva mais tempo com qualidade e autonomia.

O processo de envelhecimento varia bastante entre as pessoas e é influenciado tanto pelo estilo de vida quanto por fatores genéticos (NIEMAN, 1999). Neste, a autonomia funcional, ou também conhecida como capacidade funcional, mostra-se um dos conceitos mais relevantes em relação à saúde, aptidão física e qualidade de vida.

Para estudar esses conceitos, um grupo de pesquisadores do Laboratório de Biociências da Motricidade Humana da Universidade Castelo Branco (LABIMH), formado por docentes e discentes do curso de Mestrado desta referida instituição, fundou o Grupo de Desenvolvimento Latino-Americano para a Maturidade (GDLAM). Este se caracteriza como uma Organização de Sociedade Civil de Interesse Público (OSCIP) legalmente constituída e protocolada com o registro $n^{\circ} 11624$, em 11/05/2004.

Segundo o GDLAM, a autonomia é definida em três aspectos: autonomia de ação - referindo-se à noção de independência física; autonomia de vontade - referindo-se à possibilidade de auto-determinação; e autonomia de pensamentos - que permite ao indivíduo julgar qualquer situação. De onde pode-se concluir que autonomia não pode ser definida em apenas um aspecto, ângulo ou uma única perspectiva, mas em um contexto holístico (figura 1). Por outro lado, o mesmo grupo define independência como a capacitação de realizar tarefas sem auxílio, quer seja de pessoas, de aparelhos ou de sistemas (GDLAM, 2004).

Portanto, autonomia está associada ao declínio na habilidade para desempenhar as atividades da vida diária (AVD), e à gradual redução das funções musculares, podendo ser, conforme Posner et al. (1995), uma das principais perdas com o avançar da idade.

Recentes pesquisas apontam que indivíduos idosos podem se beneficiar dos exercícios aumentando não só a resistência e a força muscular, mas também o equilíbrio e a mobilidade. Isso pode reduzir os riscos de quedas e lesões, melhorando a autonomia funcional (ACSM, 2003; FLECK; FIGUEIRA JÚNIOR, 2003; FRONTERA; BIGARD, 2002; MATSUDO, 2002).

Para o ACSM (2003), a aptidão muscular pode tornar possível a realização das atividades da vida diária com menos esforço e prolongar a independência funcional, por permitir viver os últimos anos de modo auto-suficiente e digno.

Desta forma, o presente estudo teve como objetivo padronizar um protocolo de avaliação da autonomia funcional, denominado Protocolo GDLAM, através de uma bateria de testes relacionados com a realização das atividades da vida diária (AVD), em mulheres idosas.

\section{MATERIAIS E MÉTODOS}

\section{Amostra}

A amostra foi constituída por mulheres idosas voluntárias, aparentemente saudáveis, oriundas de grupos sociais da terceira idade,

Figura 1: Esquema da Autonomia (GDLAM, 2004)

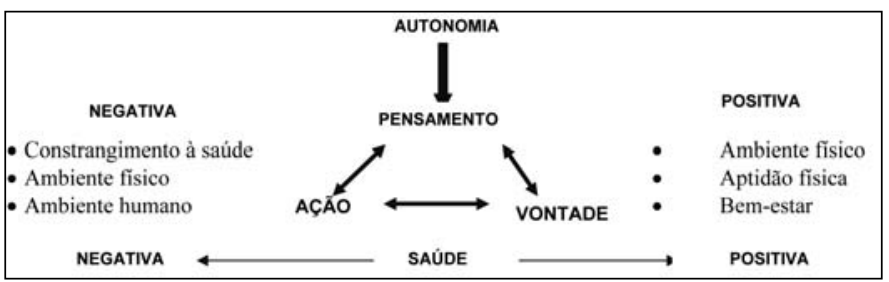


residentes na Região dos Lagos, na região do Norte Fluminense e na Zona Oeste do Estado do Rio de Janeiro e em Maceió, no Estado de Alagoas..

As voluntárias passaram por uma avaliação médica e assinaram o termo de participação consentida, de acordo com a Resolução 196/96 do Conselho Nacional de Saúde. O estudo teve seu projeto de pesquisa submetido e aprovado pelo Comitê de Ética em Pesquisa Envolvendo Seres Humanos da Universidade Castelo Branco, RJ.

Como critério de inclusão, os indivíduos da amostra deveriam ser do sexo feminino, ter idade igual ou superior a 60 anos, estarem aptas fisicamente para realizarem a bateria de testes selecionados para a avaliação da autonomia funcional, e serem independentes no desempenho das atividades físicas diárias.
Foi considerado critério de exclusão qualquer tipo de condição aguda ou crônica que pudesse comprometer ou que se tornasse um fator de impedimento para a realização dos testes.

\section{Procedimentos}

Foram utilizados os testes do protocolo de avaliação da autonomia funcional do Grupo de Desenvolvimento Latino-Americano para a Maturidade (GDLAM), constituídos de caminhar 10m (C10m), levantar-se da posição sentada (LPS), levantar-se da posição decúbito ventral (LPDV) e levantar-se da cadeira e locomover-se pela casa (LCLC). Estes testes estão descritos a seguir:

- Caminhar 10 metros $(\mathrm{C} 10 \mathrm{~m})$ - o propósito deste teste é avaliar a velocidade que o indivíduo leva para percorrer a distância de 10 metros (SIPILÄ et al., 1996) (figuras 2 e 3).
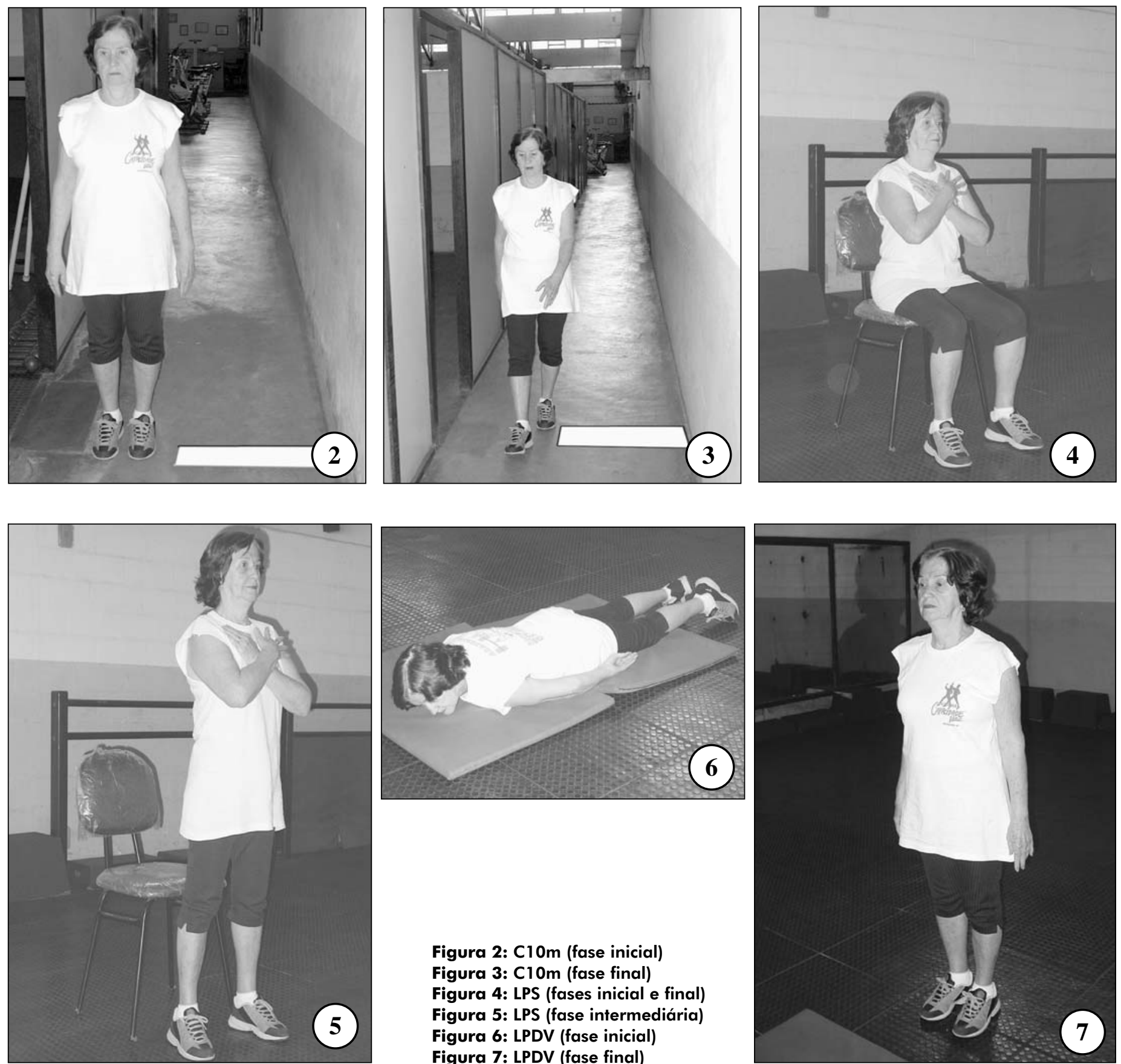

Figura 2: $\mathrm{C} 10 \mathrm{~m}$ (fase inicial) Figura 3: $\mathrm{C} 10 \mathrm{~m}$ (fase final) Figura 4: LPS (fases inicial e final) Figura 5: LPS (fase intermediária) Figura 6: LPDV (fase inicial) Figura 7: LPDV (fase final) 


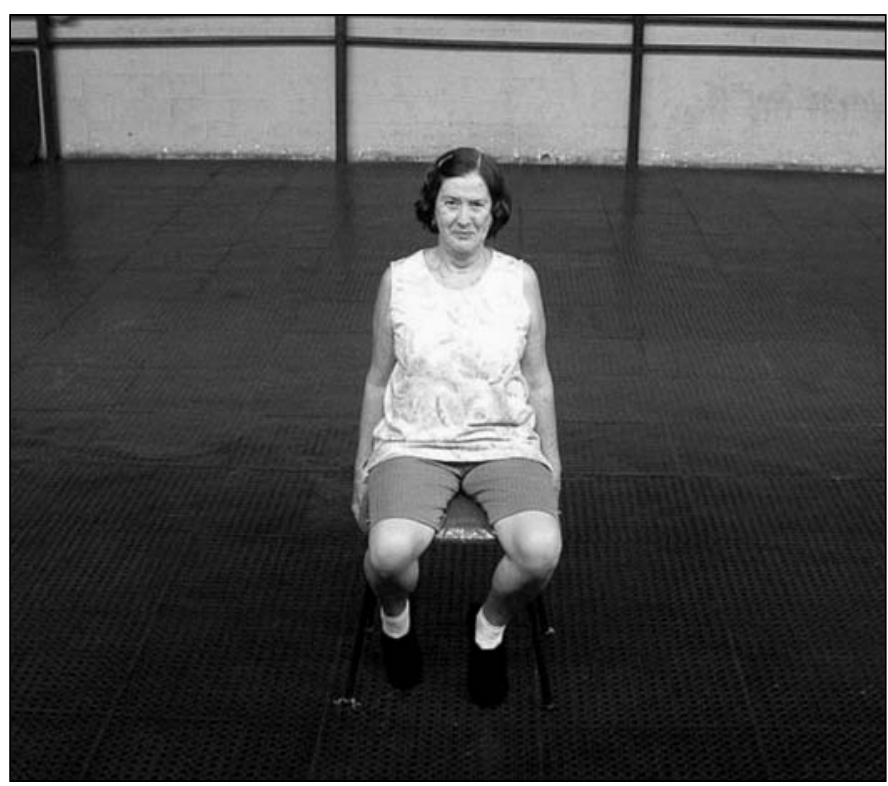

- Levantar-se da posição sentada (LPS) - o teste visa avaliar a capacidade funcional da extremidade inferior e consiste em: o indivíduo, partindo da posição sentada em uma cadeira, sem apoio dos braços, estando o assento a uma distância do solo de $50 \mathrm{~cm}$, levanta-se e senta-se cinco vezes, consecutivamente (GURALNIK et al., 1994; 1995; 2000) (figuras 4 e 5).

- Levantar-se da posição decúbito ventral (LPDV) - o propósito deste teste é avaliar a habilidade do indivíduo para levantar-se do chão. O teste consiste em: partindo da posição inicial em decúbito ventral, com os braços ao longo do corpo, ao comando de "já", o indivíduo deve levantar-se, ficando de pé o mais rápido possível (ALEXANDER et al., 1997) (figura 6 e 7).

- Levantar-se da cadeira e locomover-se pela casa (LCLC) - o objetivo é avaliar a capacidade do idoso na sua agilidade e equilíbrio, em situações da vida. Com uma cadeira fixa no solo, deve-se demarcar dois cones diagonalmente à cadeira, a uma distância de quatro metros para trás e três metros para os lados direito e esquerdo da mesma. $\bigcirc$ indivíduo inicia o teste sentado na cadeira, com os pés fora do chão, e ao sinal de "iá", ele se levanta, move-se para direita, circula o cone, retorna para a cadeira, senta-se e retira ambos os pés do chão. Sem hesitar, faz o mesmo movimento para a esquerda. Imediatamente, realiza novo percurso, para a direita e para a esquerda, assim perfazendo todo o percurso e circulando cada cone duas vezes, em menor tempo possível (ANDREOTTI; OKUMA, 1999) (figuras 8 e 9).

Os tempos destes testes foram aferidos em segundos. Os dados coletados foram analisados por meio de estatística descritiva (Média, Desvio Padrão, Quartis), através do programa "SPSS 10.0 for Windows", para estabelecer um padrão de classificação e um índice geral de autonomia (índice GDLAM - IG), conforme os tempos alcançados para a realização dos testes. $\bigcirc$ estudo admitiu o nível de $\mathrm{p}<0,05$ para a significância estatística.
O IG foi calculado por um processo de normatização entre os quatro testes de autonomia, para estimar um valor em escores. Este cálculo foi elaborado através da fórmula proposta a seguir:

$$
\mathrm{IG}=\frac{[(\mathrm{C} 10 \mathrm{~m}+\mathrm{LPS}+\mathrm{LPDV}) \times 2]+\mathrm{LCLC}}{3}
$$

onde:

C10m, LPS, LPDV e LCLC = tempo aferido em segundos.

IG = índice GDLAM em escores.

\section{INSTRUMENTOS}

Como instrumentos de avaliação dos testes, foram adotados: um cronômetro (Cásio, Malaysia), uma trena (Sanny, Brasil), um colchonete e uma cadeira com $50 \mathrm{~cm}$ de altura do assento ao solo.

\section{RESULTADOS}

Os resultados descritivos da amostra quanto às características físicas da idade e do índice de massa corporal (IMC) estão, a seguir, na tabela 1 .

Observando-se a tabela 1, verificou-se que a amostra se encontra na faixa etária do idoso (BRASIL, 1999), mas não segue uma distribuição normal quanto à variável idade. A média do IMC do grupo pesquisado apresentou um índice de classificação considerado como sobrepeso, segundo a Organização Mundial de Saúde (OMS).

Na tabela 2 estão os resultados da distribuição da normalidade pelo método de Kolmogorov-Smirnov.

Verificando-se a tabela 2, notou-se que C10m, LPS, LCLC e IG apresentaram uma distribuição próxima da normalidade. $\bigcirc$ teste LPDV não se posicionou da mesma forma, fato que não descaracteriza os dados como um todo.

Figura 9: LCLC (fase intermediária)

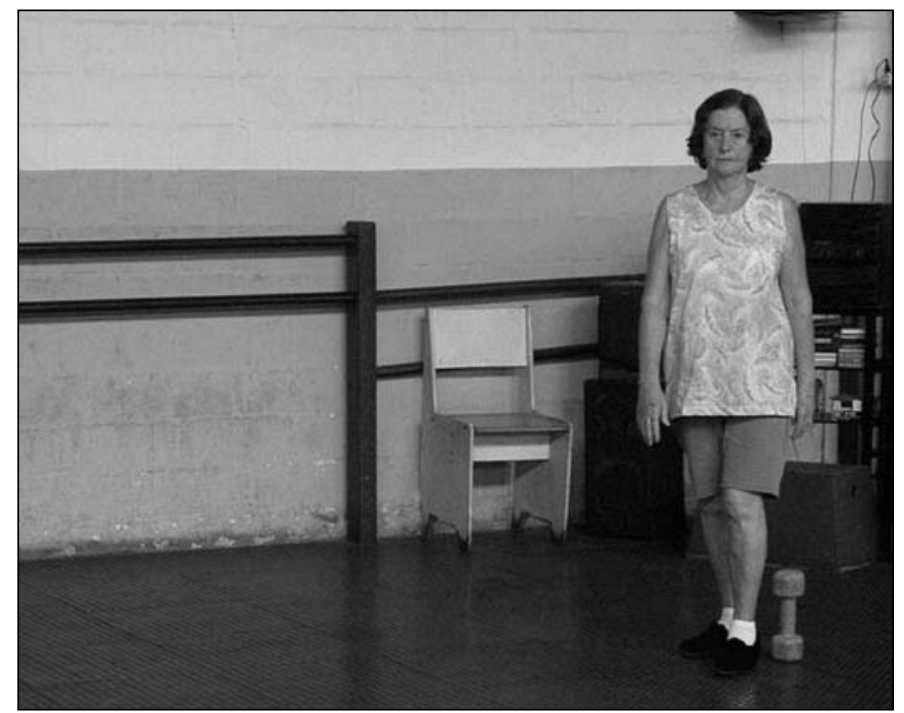


Na tabela 3 estão os resultados descritivos do protocolo GDLAM.

Analisando-se a tabela 3, observou-se que o teste LPDV foi o mais rápido para ser executado pela amostra, enquanto que o LCLC foi o mais longo. A média foi a melhor medida de tendência central para as variáveis $\mathrm{C} 10 \mathrm{~m}, \mathrm{LCLC}$ e $\mathrm{IG}$, pois o coeficiente de variação (CV) foi inferior a $20 \%$. Os valores das medianas ficaram próximos aos valores das médias. Os desvios padrão se apresentaram de forma satisfatória.

Na tabela 4 estão os resultados dos testes categorizados conforme os tempos alcançados pela amostra.

Observando-se a tabela 4, verificou-se que esta classificação pode ser um parâmetro de avaliação da autonomia do protocolo GDLAM, e ser utilizado como padrão de referência por diversos estudos nesta área de investigação.

Na tabela 5 são apresentadas as classificações da avaliação da autonomia, através do protocolo GDLAM, obtido por meio de quartis.

Analisando-se a tabela 5, verificou-se que a categorização dos tempos alcançados pela amostra, feita pelo procedimento estatístico quartil, permitiu a classificação em categorias para cada teste individualmente, e a normatização em escores do IG de autonomia, ambos em: fraco, regular, bom e muito bom.
Para a aplicabilidade desta classificação, recomenda-se o uso da média para C10m, LCLC e IG, e da mediana para os teste LPS e LPDV, conforme os resultados relativos ao coeficiente de variação - CV (tabela 3).

\section{DIsCUSSÃo}

No quadro 1 estão expostos os resultados de estudos sobre a autonomia funcional, para as atividades da vida diária (AVD).

Classificando-se os resultados dos estudos apresentados no quadro 1, de acordo com a tabela 5, verificou-se que, para 0 teste C10m, as pesquisas de Vale (2004), no grupo controle, e de Pernambuco et al. (2003) mostraram que os tempos alcançados foram considerados como fracos, enquanto trabalhos de Geraldes (2000), de Vale et al. (2003a; 2003b, 2004) e de Varejão et al. (2004) obtiveram tempos classificados em muito bom. Nos outros estudos, os resultados ficaram em classificações intermediárias.

As diferenças desses tempos podem ser justificadas pelo tipo de intervenção proposto para cada amostra. Mas os tempos alcançados são considerados satisfatórios para um idoso atravessar uma rua com segurança.

Tabela 1: Características da amostra $(n=337)$

\begin{tabular}{lccccccc}
\hline Variáveis & Média & $\mathrm{s}$ & Mediana & CV & Erro padrão & $\mathrm{a}_{3}$ & $\mathrm{a}_{4}$ \\
\hline Idade & 66,33 & 4,69 & 66,0 & $7,07 \%$ & 0,30 & 0,81 & 1,13 \\
IMC & 26,01 & 4,42 & 25,54 & $16,98 \%$ & 0,28 & 0,44 & $-0,05$ \\
\hline
\end{tabular}

$s=$ desvio padrão; $C V$ = coeficiente de variação; $a_{3}=$ assimetria; $a_{4}=$ curtose; IMC = índice de massa corporal.

Tabela 2: Resultados do teste de verificação da normalidade

\begin{tabular}{lcccc}
\hline Testes & $\mathrm{n}$ & Estatística Z & Valor-p & Distribuição \\
\hline C10m & 337 & 1,253 & 0,087 & Normal \\
LPS & 337 & 1,003 & 0,267 & Normal \\
LPDV & 313 & 2,650 & 0,000 & Não normal \\
LCLC & 92 & 0,798 & 0,548 & Normal \\
IG & 92 & 0,801 & 0,542 & Normal \\
\hline
\end{tabular}

CTOm = caminhar 10 metros; LPS = levantar da posição sentada; LPDV = levantar da posição de decúbito ventral; LCLC = levantar da cadeira e locomoverse pela casa; $I G$ = índice GDLAM.

$p<0,05$.

Tabela 3: Resultados dos testes do protocolo GDLAM

\begin{tabular}{|c|c|c|c|c|c|}
\hline Testes & $\mathrm{C} 10 \mathrm{~m}$ & LPS & LPDV & LCLC & IG \\
\hline $\mathrm{n}$ & 337 & 337 & 313 & 92 & 92 \\
\hline Média & 6,45 & 9,77 & 3,78 & 39,26 & 25,73 \\
\hline Desvio Padrão & 1,04 & 2,44 & 1,70 & 5,53 & 4,46 \\
\hline Mediana & 6,34 & 9,55 & 3,30 & 38,69 & 25,22 \\
\hline $\mathrm{CV}$ & $16,16 \%$ & $24,97 \%$ & $45,03 \%$ & $14,09 \%$ & $17,32 \%$ \\
\hline Erro padrão & 0,06 & 0,13 & 0,10 & 0,58 & 0,46 \\
\hline Mínimo & 4,03 & 4,89 & 1,64 & 30,10 & 19,02 \\
\hline Máximo & 10,84 & 17,71 & 13,33 & 58,72 & 38,23 \\
\hline Assimetria $\left(a_{3}\right)$ & 0,76 & 0,55 & 1,91 & 0,60 & 0,66 \\
\hline Curtose $\left(a_{4}\right)$ & 1,00 & 0,00 & 4,89 & 0,55 & 0,02 \\
\hline
\end{tabular}

$\mathrm{C1Om}$ = caminhar 10 metros; LPS = levantar da posição sentada; LPDV = levantar da posição de decúbito ventral; LCLC = levantar da cadeira e locomoverse pela casa.

IG = índice GDLAM em escores.

Os tempos dos testes foram aferidos em segundos. 
No teste LPS, o grupo que não sofreu intervenção do estudo de Vale (2004), o grupo que foi avaliado no início de um tratamento experimental (PERNAMBUCO et al., 2003) e o grupo do estudo de Geraldes (2000) apresentaram tempos que se localizam na classificação fraco. Achados de estudos de Baptista et al. (2003), de Baptista (2004), de Vale et al. (2003a) e de Vale (2004) mostraram tempos de execução desse referido teste no nível de classificação muito bom. Os outros resultados das demais pesquisas ficaram distribuídos nos outros níveis de classificação do Padrão do Protocolo GDLAM. O tempo marcado neste teste pode gerar uma noção da facilidade ou dificuldade que o executante idoso pode demonstrar para levantar-se de uma cadeira, que é um movimento muito freqüente na vida diária.

No teste LPDV, apenas a pesquisa de Vale (2003b) apresentou o resultado num nível de classificação muito bom. Isso pode estar relacionado com o treinamento de força que foi utilizado no tratamento experimental. Os outros estudos mostraram outras classificações. É importante ressaltar que, no estudo de Geraldes (2000), este teste foi usado na posição inicial de decúbito dorsal, diferente dos outros estudos. Esse fato proporciona um aumento do tempo de execução do mesmo, visto que o indivíduo idoso, inicialmente, gira o corpo para a posição de decúbito ventral, para depois se levantar. Este teste representa o movimento de levantar-se do chão ou de uma cama.

Notou-se que o teste LCLC foi menos utilizado nos estudos apresentados. Porém é um teste que avalia a agilidade e o equilíbrio do idoso executante, tornando-se assim um importante instrumento de avaliação da autonomia. Este teste foi o mais longo entre todos, para ser executado. Nenhum estudo mostrou um nível de classificação muito bom. Percebe-se a necessidade de avaliar a sua aplicação em mais pesquisas. Entretanto, intervenções

Tabela 4: Classificação dos tempos dos testes em quartis

\begin{tabular}{lcccc}
\hline Testes & $1^{\circ}$ Quartil & $2^{\circ}$ Quartil & $3^{\circ}$ Quartil & $4^{\circ}$ Quartil \\
\hline C10m & $>7,09$ & $7,09-6,34$ & $6,33-5,71$ & $<5,71$ \\
LPS & $>11,19$ & $11,19-9,55$ & $9,54-7,89$ & $<7,89$ \\
LPDV & $>4,40$ & $4,40-3,30$ & $3,29-2,63$ & $<2,63$ \\
LCLC & $>43,00$ & $43,00-38,69$ & $38,68-34,78$ & $<34,78$ \\
\hline
\end{tabular}

C10m = caminhar 10 metros; LPS = levantar da posição sentada; LPDV = levantar da posição de decúbito ventral; LCLC = levantar da cadeira e locomoverse pela casa.

A distribuição dos tempos dos testes foi feita em segundos.

Tabela 5: Padrão de Avaliação da Autonomia Funcional do Protocolo GDLAM

\begin{tabular}{lccccc}
\hline Testes Classif. & $\begin{array}{c}\text { C10m } \\
(\mathrm{seg})\end{array}$ & $\begin{array}{c}\text { LPS } \\
(\mathrm{seg})\end{array}$ & $\begin{array}{c}\text { LPDV } \\
(\mathrm{seg})\end{array}$ & $\begin{array}{c}\text { LCLC } \\
(\mathrm{seg})\end{array}$ & $\begin{array}{c}\text { IG } \\
\text { (escores) }\end{array}$ \\
\hline Fraco & $+7,09$ & $+11,19$ & $+4,40$ & $+43,00$ & $+28,54$ \\
Regular & $7,09-6,34$ & $11,19-9,55$ & $4,40-3,30$ & $43,00-38,69$ & $28,54-25,25$ \\
Bom & $6,33-5,71$ & $9,54-7,89$ & $3,29-2,63$ & $38,68-34,78$ & $25,24-22,18$ \\
Muito Bom & $-5,71$ & $-7,89$ & $-2,63$ & $-34,78$ & $-22,18$ \\
\hline
\end{tabular}

$\mathrm{C} 10 \mathrm{~m}$ = caminhar 10 metros; LPS = levantar da posição sentada; LPDV = levantar da posição de decúbito ventral; LCLC = levantar da cadeira e locomoverse pela casa; $I G$ = índice GDLAM.

Quadro 1: Estudos sobre autonomia das AVD, através do Protocolo GDLAM

\begin{tabular}{lccccc}
\hline Estudo (ano) & Treinamento & C10m & LPS & LPDV & LCLC \\
\hline Amorim, 2002 & Aeróbico & 6,78 & 10,68 & 3,88 & - \\
Aragão, 2002 & RML & 7,0 & 11,0 & 4,5 & - \\
Baptista et al., 2003 & Yoga & 6,26 & 7,55 & 2,92 & - \\
Baptista, 2004 & Yoga & 6,97 & 7,34 & 2,71 & - \\
Geraldes, 2000 & Força & 5,6 & 11,4 & $4,1^{*}$ & - \\
Pernambuco et al., 2003 & Shiatsu & 7,46 & 12,95 & 5,73 & 54,12 \\
Pernambuco, 2004 & Shiatsu & 6,07 & 10,11 & 3,21 & 39,17 \\
Vale et al., 2003a & Flex. Dinâmico & 5,93 & 8,35 & 3,35 & 38,72 \\
Vale et al., 2003b & Flex. Dinâmico & 5,44 & 7,70 & 2,90 & - \\
Vale, 2004 & Força & 5,35 & 8,30 & 2,54 & - \\
& Força 75-85\% & 5,65 & 7,16 & 3,28 & 35,76 \\
Varejão et al., 2004 & Flex. Dinâmico & 5,93 & 8,35 & 3,32 & 38,35 \\
& Controle & 7,11 & 13,23 & 4,99 & 46,31 \\
\hline
\end{tabular}

C10m = caminhar 10 metros; LPS = levantar da posição sentada; LPDV = levantar da posição de decúbito ventral; LCLC = levantar da cadeira e locomoverse pela casa. Média=tempo (segundos).

* O teste realizado foi em decúbito dorsal. Tempo em segundos. 

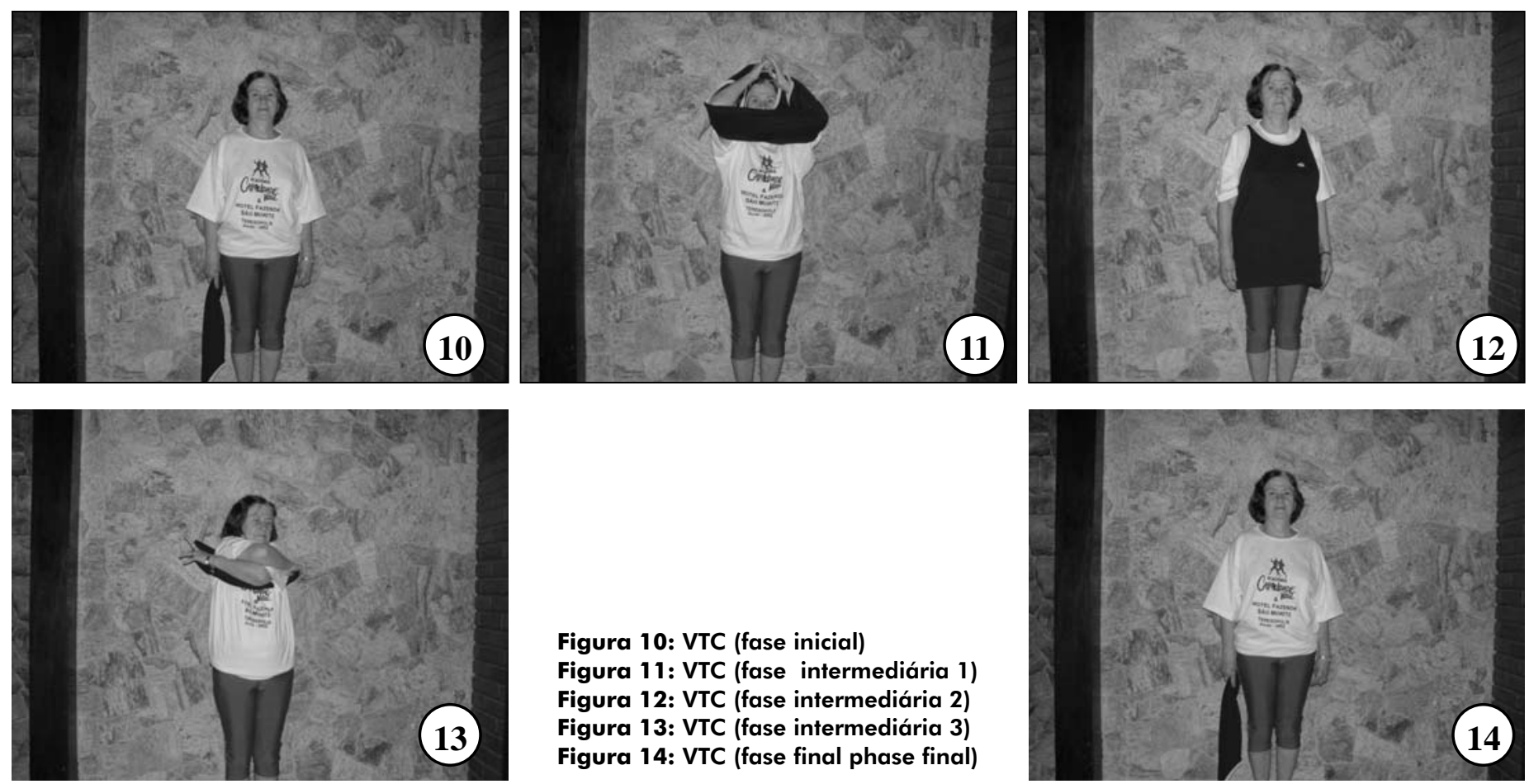

Figura 10: VTC (fase inicial)

Figura 11: VTC (fase intermediária 1)

Figura 12: VTC (fase intermediária 2)

Figura 13: VTC (fase intermediária 3)

Figura 14: VTC (fase final phase final)

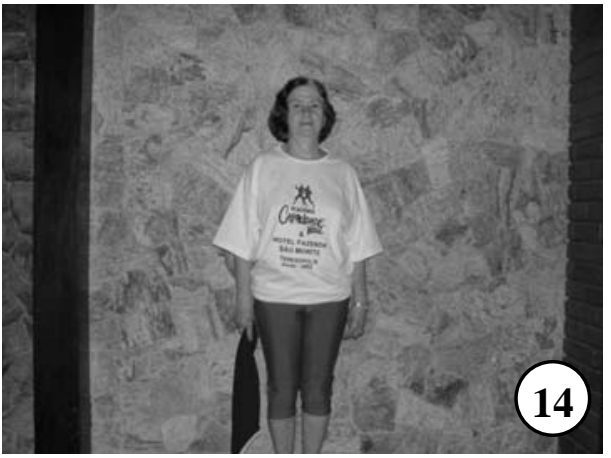

que exigiram trabalhos de força obtiveram melhores resultados (VALE, 2004).

De uma maneira geral, ao analisar o quadro 1, notou-se que os sujeitos idosos sedentários (PERNAMBUCO et al., 2003; VALE, 2004) obtiveram tempos que estão situados num nível de classificação fraco, do Padrão do Protocolo GDLAM, para todos os testes realizados. Isso indica que os idosos que permanecerem inativos fisicamente ao longo da vida deverão sofrer os efeitos do envelhecimento com maior impacto, entretanto aqueles que se mantiverem ativos fisicamente tenderão a prolongar a autonomia funcional e a qualidade de vida.

Considerando-se que a autonomia funcional está associada às atividades da vida diária (AVD), o índice geral (IG) foi idealizado para representar o nível desta variável nos indivíduos idosos. Devido à natureza dos movimentos e sua relação ao cotidiano, o conjunto dos testes realizados parece permitir uma visão global do geronte.

Conforme os resultados apresentados e a metodologia aplicada, o presente estudo sugere a criação de mais um teste para a avaliação da autonomia funcional, que esteja relacionado com os movimentos dos membros superiores. Aponta-se então o teste de vestir e tirar uma camiseta (VTC), onde o tempo de realização do mesmo será marcado em segundos (figuras 10, 11, 12, 13 e 14). Quanto menor o tempo de execução, melhor será o resultado. indivíduo deve estar de pé, com os braços ao longo do corpo e com uma camiseta em uma das mãos. Ao sinal de "já", ele deve vestir a camiseta e, imediatamente, retirá-la, retornando à posição inicial. Este teste visa avaliar a agilidade e a coordenação dos membros superiores.

Acredita-se que, com a inclusão do VTC, o Padrão do Protocolo GDLAM de autonomia funcional ficará mais completo em sua avaliação global. Isso demonstra a tentativa de se estabelecer um teste que se propõe avaliar um movimento que tende a apresentar dificuldade de realização com o envelhecimento, e que se enquadra nas AVD.

\section{CONCLUSÃO}

De acordo com os achados na presente pesquisa, o IG pode ser utilizado como parâmetro da avaliação da autonomia funcional, para a realização das AVD. Da mesma forma, o estudo indica que o Padrão do Protocolo GDLAM pode ser aplicado como referência para futuras investigações, e que se introduza o VTC para que se investiguem novos padrões de referência da autonomia.

\section{AGRADECIMENTOS}

Agradecimentos aos Professores Mestres da linha de pesquisa "Atividade Física, Autonomia e Qualidade de Vida do Idoso" do PROCIMH - UCB/RJ, Jani Aragão, Lilliany Cordeiro, Márcio Baptista, Carlos Pernambuco, Fátima Amorim, Amândio Geraldes e Ronaldo Varejão, que gentilmente cederam os dados de suas respectivas dissertações de mestrado, para a elaboração deste estudo.

\section{REFERÊNCIAS}

ALEXANDER, Neil B.; ULBRICH, Jessica; RAHEJA, Aarti; CHANNER, Dwight. Rising from the floors in older adults. Journal of the American Geriatrics Society. v. 45, $n$. 5, p. 564-569, 1997.

AMERICAN COLLEGE OF SPORTS MEDICINE. Diretrizes do ACSM para os testes de esforço e sua prescrição. 6a edição. Rio de Janeiro: Guanabara Koogan, 2003.

AMORIM, Fátima $S$. Efeitos do treinamento da capacidade aeróbica sobre a qualidade de vida e autonomia de idosos. 2002, 231 f. Dissertação (Mestrado em Ciência da Motricidade Humana). Universidade Castelo Branco - UCB. Rio de Janeiro. 
ANDREOTTI, Rosana A.; OKUMA, Silene S. Validação de uma bateria de testes de atividades da vida diária para idosos fisicamente independentes. Revista Paulista de Educação Física, v. 13, n. 1, p. 46-66, 1999.

ARAGÃO, Jani Cleria Bezerra de. Efeitos da resistência muscular localizada visando a autonomia e a qualidade de vida de idosos. 2002, $332 \mathrm{f}$. Dissertação (Mestrado em Ciência da Motricidade Humana). Universidade Castelo Branco - UCB. Rio de Janeiro.

BAPTISTA, Márcio Rodrigues. A prática do yoga sobre a autonomia funcional e qualidade de vida em mulheres senescentes. 2004, 325 f. Dissertação (Mestrado em Ciência da Motricidade Humana). Universidade Castelo Branco - UCB. Rio de Janeiro.

BAPTISTA, Marcio R.; VALE, Rodrigo G. S.; PERNAMBUCO, Carlos S.; DANTAS, Estélio H. M. O yoga na autonomia funcional em mulheres senescentes. In: XXVI SIMPÓSIO INTERNACIONAL DE CIÊNCIAS DO ESPORTE, São Paulo, 2003. Anais: Atividade física construindo saúde. Edição esp. Revista Brasileira de Ciência e Movimento, p. 82, 2003.

BRASIL. Ministério da Saúde. Resolução 196/96. O Plenário do Conselho Nacional de Saúde resolve aprovar diretrizes e normas regulamentadoras de pesquisas envolvendo seres humanos. Em 10 de outubro de 1996.

BRASIL. Ministério da Saúde. Portaria n 1395/GM. Dispõe sobre a POLÍTICA NACIONAL DE SAÚDE DO IDOSO. Em 10 de dezembro de 1999.

FLECK, S. J.; FIGUEIRA JÚNIOR, A. Treinamento de força para fitness e saúde. São Paulo: Phorte Editora, 2003.

FRONTERA, W. R.; BIGARD, X. The benefits of strength training in the elderly. Science and Sports, v. 17, i 3, p. 109-116, May, 2002.

GERALDES, A. A. R. Efeitos do treinamento contra resistência sobre a força muscular e o desempenho de habilidades funcionais selecionadas em mulheres. 2000, $214 \mathrm{f}$. Dissertação (Mestrado em Ciência da Motricidade Humana), Universidade Castelo Branco, UCB. Rio de Janeiro.

GRUPO DE DESENVOLVIMENTO LATINO-AMERICANO PARA MATURIDADE (GDLAM). Discussões de estudo: conceitos de autonomia e independência para o idoso. Rio de Janeiro, 2004.

GURALNIK, Jack M.; SIMONSICK, Eleanor M.; FERRUCCI, Liugi; GLYNN, Robert J.; BERKMAN, Lisa F.; BLAZER, Dan G.; SCHERR, Paul A.; WALLACE, Robert B. A short physical performance battery assessing lower extremity function: association with self-reported disability and prediction of mortality and nursing home admission. The Journal of Gerontology, v. 49, n. 2, p. M85-M94, 1994.

GURALNIK, Jack M.; FERRUCCI, Liugi; SIMONSICK, Eleanor M.; SALIVE, Marcel E. WALLACE, Robert B. Lower-extremity function in persons over the age of 70 years as a predictor of subsequent disability. The New England Journal of Medicine. v. 332, n. 9, p. $556-561,1995$.

GURALNIK, Jack M.; FERRUCCI, Liugi; PIEPER, C. F.; LEVEILLE, S. G.; MARKIDES, K. S.; OSTIR, G. V.; STUDENSKI, S.; BERKMAN, L. F.; WALLACE, Robert B. Lower extremity function and subsequent disability consistency across studies, predictive models and value of gait speed alone compared with the short physical performance battery. Journal of Gerontology. v. 55, n. 4, p. M221-M231, 2000.

INSTITUTO BRASILEIRO DE GEOGRAFIA E ESTATíSITICA (IBGE). Base de dados, censo 2000. Disponível na Internet em: <http://www.ibge.gov.br> Acesso em $10 \mathrm{de}$ março de 2003.

MATSUDO, S. M. M. Envelhecimento, atividade física e saúde. Revista Mineira de Educação Física, v. 10, n. 1, p. 193-207, 2002

NIEMAN, D. C. Exercício e saúde. $1^{a}$ edição. São Paulo: Manole, 1999.

PERNAMBUCO, Carlos S.; VALE, Rodrigo G. S.; BAPTISTA, Marcio R.; ABREU, Flávia M. C.; DANTAS, Estélio H. M. Perfil da autonomia funcional de idosos no ingresso de um programa de shiatsuterapia no município de Araruama. In: XXVI SIMPÓSIO INTERNACIONAL DE CIÊNCIAS DO ESPORTE, São Paulo, 2003. Anais: Atividade física construindo saúde. Edição esp. Revista Brasileira de Ciência e Movimento, p. 78, 2003.

PERNAMBUCO, Carlos S. Comparação de um programa de shiatsuterapia e de um programa de flexionamento dinâmico na flexibilidade, na autonomia e qualidade de vida do idoso. 2004, 177 f. Dissertação (Mestrado em Ciência da Motricidade Humana). Universidade Castelo Branco - UCB. Rio de Janeiro.

POSNER, Joel D.; McCULLY, Kevin K.; LANDSBERG, Lisa A.; SANDS, Laura P.; TYCENSKI, Patricia; HOLFMANN, Mary T.; WETTERHOLT, Kristina L.; SHAW, Carl E. Physical determinants of independence in mature women . Archive of Physical Medicine and Rehabilitation, v. 76, p. 373-380, 1995.

SIPILÄ, S.; MULTANEN, J.; KALLINEN, M.; ERA, P.; SUOMINEN, H. Effects of strength and endurance training on isometric muscle strength and walking speed in elderly women. Acta Physiologica Scandinavica, v. 156, p. 457-464, 1996.

VALE, R. G. S.; ARAGÃO, J. C. B. - DANTAS, E. H. M. A flexibilidade na autonomia funcional de idosas independentes. Fitness e Performance Journal, v. 2, n. 1, p. 23-29, 2003a.

VALE, Rodrigo G. S.; BAPTISTA, Marcio R.; PERnAMBUCO, Carlos S.; VIEIRA, Fernando R.; ARAGÃO, Jani C. B.; DAMASCENO, Vinicius; CORDEIRO, Lilliany S.; NOVAES, Jefferson S.; DANTAS, Estélio H. M. Treinamento resistido de força em idosas independentes. In: XXVI SIMPÓSIO INTERNACIONAL DE CIÊNCIAS DO ESPORTE, São Paulo, 2003. Anais: Atividade física construindo saúde. Edição esp. Revista Brasileira de Ciência e Movimento, p. 53, $2003 \mathrm{~b}$.

VALE, Rodrigo G. S. Efeitos do treinamento de força e de flexibilidade sobre a autonomia e qualidade de vida de mulheres senescentes. 2004, $232 \mathrm{f}$. Dissertação (Mestrado em Ciência da Motricidade Humana). Universidade Castelo Branco - UCB. Rio de Janeiro.

VAREJÃO, Ronaldo V.; MELO, Roberto; BARROS, Rosilane; VALE, Rodrigo G. S.; ARAGÃO, Jani C. B.; AMORIM, Fátima S.; DANTAS, Estélio H. M. Comparação dos efeitos do alongamento e do flexionamento ambos passivos sobre os níveis de flexibilidade, autonomia e qualidade de vida do idoso. FIEP Bulletin, v. 74, 2004. 